\title{
A New Two-Parameter Heteromorphic Elliptic Equation: Properties and Applications
}

\author{
Zhouhu Wu \\ School of Environmental and Municipal Engineering, Qingdao University of Technology, Qingdao, China \\ Email:wu_zh2008@qut.edu.cn
}

How to cite this paper: Wu, Z.H. (2020) A New Two-Parameter Heteromorphic Elliptic Equation: Properties and Applications. World Journal of Engineering and Technology, 8, 642-657. https://doi.org/10.4236/wjet.2020.84045

Received: April 8, 2020

Accepted: October 28, 2020

Published: November 4, 2020

\begin{abstract}
The ellipse and the superellipse are both planar closed curves with a double axis of symmetry. Here we show the isoconcentration contour of the simplified two-dimensional advection-diffusion equation from a stable line source in the center of a wide river. A new two-parameter heteromorphic elliptic equation with a single axis of symmetry is defined. The values of heights, at the point of the maximum width and that of the centroid of the heteromorphic ellipse, are derived through mathematical analysis. Taking the compression coefficient $\theta=b / a=1$ as the criterion, the shape classification of H-type, Standard-type and $\mathrm{W}$-type for heteromorphic ellipse have been given. The area formula, the perimeter theorem, and the radius of curvature of heteromorphic ellipses, and the geometric properties of the rotating body are subsequently proposed. An illustrative analysis shows that the inner contour curve of a heteromorphic elliptic tunnel has obvious advantages over the multiplearc splicing cross section. This work demonstrates that the heteromorphic ellipses have extensive prospects of application in all categories of tunnels, liquid transport tanks, aircraft and submarines, bridges, buildings, furniture, and crafts.
\end{abstract}

\section{Keywords}

Two-Parameter Curve, Heteromorphic Ellipse, Standard Equation, Geometric Properties, Engineering Applications

\section{Introduction}

The ellipse is a common planar closed curve with a double axis of symmetry, belonging to the family of conic curves represented by a quadratic equation [1]. The general Cartesian notation for the form comes from the French mathematician Gabriel Lamé, who generalized the equation for the ellipse. A superelliptic 
equation (i.e., $|x / a|^{n}+|y / b|^{n}=1$, where $n, a$, and $b$ are positive numbers) is a closed curve resembling the ellipse with three parameters. A superellipse, similarly to the ellipse, is symmetric about its two semi-axes, but differs in its overall shape [2] [3]. The Danish author and scientist Piet Hein have dealt with the superellipse in great detail, especially by using the curve for architectural objects such as motorway bridges [4]. His proposal won the design challenge for a roundabout in the Sergels Torg city square in Stockholm, Sweden. The Melior font, designed by Hermann Zapf, has been based on this curve [3]. The egg curves (i.e., $[x / a]^{2}+[y / b]^{2} t(x)=1$, where $t(x)$ is a function of $x$ ) have been discovered by Florian Blaschke [5]. An oval is a closed plane line that is shaped like an ellipse or like the egg of a hen. The hen's egg is smaller at one end and has only one axis of symmetry [6]. Reference [7] egg-shape equation (i.e., $[x / a]^{2}+[y /(k y+b)]^{2}=$ $1,|k|<1)$ have three parameters. These can develop the shape of a hen egg, which changes the equation of an oval a little. As important mathematical tools, these curvilinear equations play an important role in daily production and life.

This study is based on the isoconcentration contour equation of simplified two-dimensional advection-diffusion in rivers. The scope of this study encompass 1) the analysis of the height in the direction of the axis of symmetry and the width in the direction of asymmetry, 2) determine a new equation for a heteromorphic elliptic equation with two parameters, 3) compare the heteromorphic ellipse with an ellipse, and 4) investigate the geometric properties and application prospects of the heteromorphic ellipse and the rotating body. The results can provide theoretical support for the popularization and application of the heteromorphic elliptic equation.

\section{Background of the Heteromorphic Elliptic Equation}

Reference [8] has given the concentration distribution from a uniform-intensity line source in the center of a wide river based on the simplified two-dimensional advection-diffusion transport equation. The resulting equation is given as

$$
C(x, y)=\frac{m}{H \sqrt{4 \pi E_{y} U x}} \exp \left(-\frac{U y^{2}}{4 E_{y} x}\right)
$$

where $x$ is the longitudinal coordinate in the stream-wise direction, and $y$ is the transverse coordinate perpendicular to the flow and pointing towards the river bank. The origin of the coordinate system is the discharge point at the center of the river, $m$ the mass discharge rate of the passive scalar, $U$ the average velocity of the river, $H$ the average water depth, and $E_{\mathrm{y}}$ the transverse diffusion coefficient.

According to Equation (1), for a constant concentration $C=C_{\mathrm{a}}$, the maximum length $L_{\mathrm{s}}$, the maximum half-width $b_{\mathrm{s}}$, and the corresponding longitudinal coordinate $L_{\mathrm{c}}$ of the area surrounded by the contour line are given as [9]

$$
L_{s}=\frac{1}{4 \pi E_{y} U}\left(\frac{m}{H C_{a}}\right)^{2}
$$




$$
\begin{gathered}
b_{s}=\sqrt{\frac{2 E_{y} L_{s}}{\mathrm{e} U}} \\
L_{c}=\frac{L_{s}}{\mathrm{e}}
\end{gathered}
$$

Wu et al. [10] have used $L_{\mathrm{s}}$ and $b_{\mathrm{s}}$ in Equation (2) and Equation (3) to make $x$ and $y$ dimensionless in Equation (1) with $C(x, y)=C_{\mathrm{a}}$ and obtained the dimensionless formula of the isoconcentration curve (namely Wu's curve) as

$$
\left(\frac{y}{b_{s}}\right)^{2}=-\mathrm{e} \frac{x}{L_{s}} \ln \left(\frac{x}{L_{s}}\right)
$$

Previous studies have indicated that Wu's curve as represented by Equation (5) is similar to the ellipse, whereas it has only a single axis of symmetry. Since it is derived from advection and diffusion of a passive scalar and the transport of momentum, heat, and mass have certain similarities in flow, the authors have surmised that Wu's curve could exhibit good mechanical properties analogous to that of the shape of the eggshell, and hence enhancing the significance of the Wu's curve. For the sake of describing this metaphysical concept by a representational image, Wu's curve is referred to here as a "heteromorphic ellipse".

\section{Comparison of the Heteromorphic Ellipse with the Ellipse}

Following Equation (5), the heteromorphic ellipse has its maximum dimension along the axis of symmetry in the $x$-direction. Hereafter, for simplicity, the scale in the $x$ and $y$ axes will be called "height" and "width", respectively. Defining such a length scale as the maximum height of the heteromorphic ellipse, $2 a=L_{\mathrm{s}}$, and the maximum width as $2 b=2 b_{s}$, the new equation of the heteromorphic ellipse as shown in Figure 1 can be obtained using Equation (5) as

$$
\left(\frac{y}{2 b}\right)^{2}=-\frac{\mathrm{e}}{4} \frac{x}{2 a} \ln \left(\frac{x}{2 a}\right),(a>0, b>0)
$$

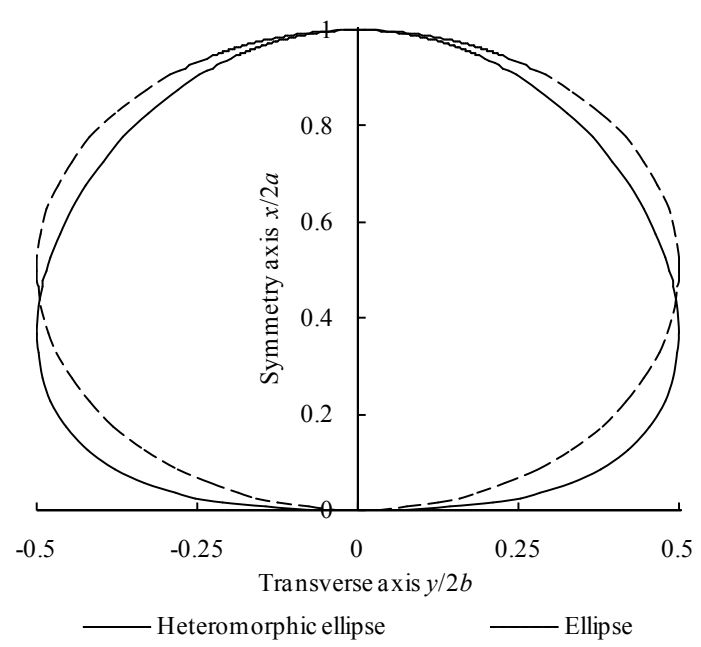

Figure 1. Comparison of the heteromorphic ellipse and an ellipse with the same long and short axes. 
The median line of the heteromorphic ellipse is located at the midpoint of the axis of symmetry and it is a line segment that connects two side boundary points that is parallel to the asymmetric axis. Alternatively, the height of the median line of the heteromorphic ellipse is $h=a$. From Equation (4), it can be concluded that the maximum width is located at the height $L_{\mathrm{c}} / 2 a=1 / \mathrm{e} \approx 0.368$. This value is $26.42 \%$ less than the median line relative height.

It divides the whole geometry into upper and lower parts at the approach to the golden section of the line. The heteromorphic ellipse is symmetrical about the $x$-axis and ranges from $0 \leq x \leq 2 a$ and $-b \leq y \leq b$. The four vertices at the bottom, top, left, and right boundaries are at $(0,0),(2 a, 0),(1 / e,-b)$ and $(1 / e, b)$, respectively (Figure 1). The heteromorphic ellipse looks like a deformed ellipse. Figure 1 also compares the heteromorphic ellipse with an ellipse of the same size along the long and short axes and centered at $x / 2 a=0.5, y / 2 b=0$.

The two vertices on the axis of symmetry $x$ are coincident, with the same height and width, in the heteromorphic ellipse and the ellipse (Figure 1). On each side of the symmetric $x$-axis, the heteromorphic ellipse intersects the ellipse at $x / 2 a \approx 0.436, y / 2 b \approx \pm 0.496$. Above these intersections, the heteromorphic ellipse is narrower than the ellipse, whereas below the intersections, it is wider. Alternatively, the heteromorphic ellipse presents certain distinguishing features, viz. wide lower part, flat base, narrow upper part, and slightly pointed top. These geometric features lead to a lower centroid with obvious advantages for upright stability.

\section{Geometric Properties of the Heteromorphic Ellipse}

\subsection{Area}

The integral of Equation (6) is determined on $x \in[0,2 a]$, the formula for calculating the area of the heteromorphic ellipse be derived as

$$
S=2 \int_{0}^{2 a} 2 b \sqrt{-\frac{\mathrm{e}}{4} \frac{x}{2 a} \ln \left(\frac{x}{2 a}\right)} \mathrm{d} x=4 a b \sqrt{\mathrm{e}} \int_{0}^{2 a} \sqrt{-\frac{x}{2 a} \ln \left(\frac{x}{2 a}\right)} \mathrm{d}\left(\frac{x}{2 a}\right)
$$

By performing variable substitution so that $x / 2 a=\zeta$, the upper limit of the integral becomes one, and the result is given as

$$
S=4 a b \sqrt{\mathrm{e}} \int_{0}^{1} \sqrt{-\zeta \ln (\zeta)} \mathrm{d} \zeta=\mu(2 a)(2 b)
$$

where $\mu=\sqrt{\mathrm{e}} \int_{0}^{1} \sqrt{-\zeta \ln (\zeta)} \mathrm{d} \zeta$ is called the "area coefficient". Hence, the area formula for the heteromorphic ellipse states that its area is equal to the product of the area coefficient, the height, and the width.

Let $\eta=\zeta^{.5}$, and by substituting the area coefficient formula, the definite integral can be obtained directly that the area coefficient can be given as

$$
\mu=\frac{2}{3} \sqrt{\mathrm{e}} \int_{0}^{1} \sqrt{\frac{2}{3} \ln \left(\zeta^{-\frac{3}{2}}\right)} \mathrm{d} \zeta^{\frac{3}{2}}=\left(\frac{2}{3}\right)^{1.5} \sqrt{\mathrm{e}} \int_{0}^{1} \sqrt{\ln \left(\eta^{-1}\right)} \mathrm{d} \eta
$$

An integral table can be used to evaluate $\int_{0}^{1} \sqrt{\ln \left(\eta^{-1}\right)} \mathrm{d} \eta=\sqrt{\pi} / 2$, which is substituted into Equation (9) to give 


$$
\mu=\left(\frac{2}{3}\right)^{1.5} \frac{\sqrt{\pi \mathrm{e}}}{2} \approx 0.795
$$

This value is $1.27 \%$ larger than the area coefficient $(=\pi / 4)$ of the ellipse.

\subsection{Centroid Coordinate}

The centroid refers to the geometric center of a heteromorphic ellipse, and for objects of uniform density, the center of mass and the centroid are coincident. For a heteromorphic ellipse with only one axis of symmetry, the centroid must be on its axis of symmetry. To identify the specific point on the axis of symmetry, it is necessary to determine the area moment by calculating the area moments for the asymmetric axis. According to the principle that the total area moment is equal to the sum of each area moment

$$
S_{m} \cdot x_{\mathrm{c}}=\int_{0}^{S} x \mathrm{~d} S=16 a^{2} b \int_{0}^{2 a} \frac{x}{2 a} \sqrt{-\frac{\mathrm{e}}{4} \frac{x}{2 a} \ln \left(\frac{x}{2 a}\right)} \mathrm{d}\left(\frac{x}{2 a}\right)
$$

Using variable substitution, letting $x / 2 a=\zeta$, and setting $\eta=\zeta^{2.5}$, the upper limit of the integral is constant, and the upper limit of the integral becomes one. By substituting this into Equation (11), the centroid coordinate of the heteromorphic ellipse can be obtained as

$$
x_{\mathrm{c}}=\frac{2 a \sqrt{\mathrm{e}}}{\mu_{m}}\left(\frac{2}{5}\right)^{1.5} \int_{0}^{1} \sqrt{\ln \left(\zeta^{-\frac{5}{2}}\right)} \mathrm{d} \zeta^{\frac{5}{2}}=\frac{2 a \sqrt{\mathrm{e}}}{\mu_{m}}\left(\frac{2}{5}\right)^{1.5} \int_{0}^{1} \sqrt{\ln \left(\eta^{-1}\right)} \mathrm{d} \eta
$$

Using an integral table to evaluate $\int_{0}^{1} \sqrt{\ln \left(\eta^{-1}\right)} \mathrm{d} \eta=\sqrt{\pi} / 2$ and then substituting Equation (10) into Equation (12), we get

$$
x_{\mathrm{c}}=\frac{a \sqrt{\pi \mathrm{e}}}{\mu_{m}}\left(\frac{2}{5}\right)^{1.5}=\left(\frac{3}{5}\right)^{1.5}(2 a)=0.465(2 a)
$$

Therefore, the centroid of a heteromorphic ellipse is located at the point on the axis of symmetry where the relative height coordinate is $x_{\mathrm{c}}{ }^{\prime}=x_{\mathrm{c}} / 2 \mathrm{a}=0.465$. The height of this point is $7.05 \%$ lower than the median line (ellipse centroid) height, and for uniform-mass objects, the centroid of the heteromorphic ellipse is lowered by $7.05 \%$. Hence, the vertical stability of a heteromorphic ellipse is better than that of an ellipse.

\subsection{Compression Coefficient and Shape Classification}

The compression coefficient of a heteromorphic ellipse is defined as the ratio of the width of the asymmetric axis and the height of the axis of symmetry (the ratio of width to height being $\theta=2 b / 2 a=b / a(\theta>0))$. Figure 2 shows certain heteromorphic ellipse shapes with $\theta=0.25,0.67,1.00$, and 1.50 .

Under different compression coefficients, the shape of a heteromorphic ellipse seems to follow natural patterns (Figure 2). The shapes of the rotating body of these figures are as follows: Figure 2(a) takes the shape of a corn cob, Figure 2(d) that of round bread, and Figure 2(b) and Figure 2(c) resemble the shape of strawberry and apple, etc. 


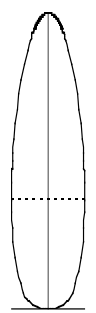

(a)

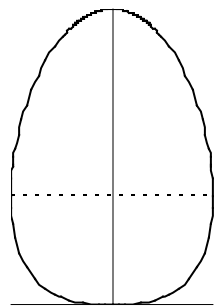

(b)

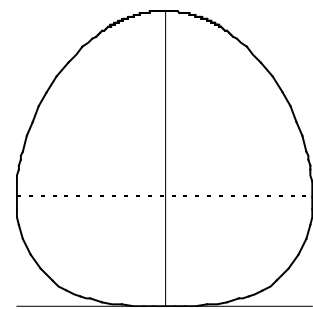

(c)

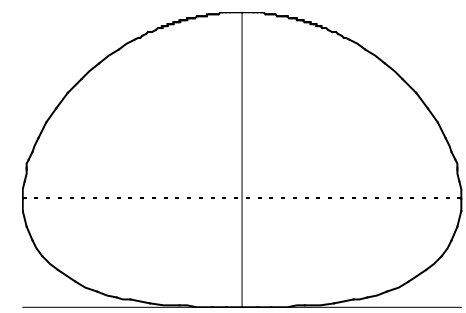

(d)

Figure 2. Heteromorphic ellipses with different compression coefficients. (a) $\theta=0.25$; (b) $\theta=0.67$; (c) $\theta=1.00$; (d) $\theta=1.50$.

To note, the compression coefficient can reflect the shape characteristics of the heteromorphic ellipse. Accordingly, the shape of the heteromorphic ellipse can be classified for the cases of: 1) $0<\theta<1$, where the width of the heteromorphic ellipse is less than the height, and the ellipse is of leptosome type (H-type), 2) $\theta=1$, where the width of the heteromorphic ellipse is equal to the height, which is called the Standard-type, 3) $\theta>1$, where the width of the heteromorphic ellipse is greater than the height, and the ellipse is of pyknic type (W-type).

Practical experience reveals that as compared with ellipses with a double axis of symmetry, whose shapes have only one axis of symmetry with one end thin and the other end having a large oval shape, are more common in nature.

\subsection{Perimeter}

According to the integral of the plane curve arc length in higher mathematics, the perimeter integral formula of a heteromorphic ellipse is given as

$$
L=2 \int_{0}^{2 a} \sqrt{1+y^{\prime 2}} \mathrm{~d} x
$$

Taking the derivative on both sides of Equation (6) results in the following

$$
y^{\prime}=-\frac{\sqrt{\mathrm{e}}}{4} \frac{b}{a}\left[1+\ln \left(\frac{x}{2 a}\right)\right] / \sqrt{-\frac{x}{2 a} \ln \left(\frac{x}{2 a}\right)}
$$

Substituting Equation (15) into Equation (14), setting $x / 2 a=\zeta$, and noting that $b / a=\theta$, the upper limit of the integral becomes 1 , and the integral formula for the heteromorphic elliptic perimeter is given as

$$
L=\frac{4}{1+\theta} \int_{0}^{1} \sqrt{1-\frac{\mathrm{e} \theta^{2}}{16} \frac{(1+\ln \zeta)^{2}}{\zeta \ln \zeta}} \mathrm{d} \zeta(a+b)=T_{h}(a+b)
$$

where $T_{h}=\frac{4}{1+\theta} \int_{0}^{1} \sqrt{1-\frac{\mathrm{e} \theta^{2}}{16} \frac{(1+\ln \zeta)^{2}}{\zeta \ln \zeta}} \mathrm{d} \zeta$ is called the "heteromorphic elliptic coefficient". The theorem regarding the perimeter of a heteromorphic ellipse can be stated as, the perimeter is equal to the product of the heteromorphic elliptic coefficient and the sum of the half-height and half-width of the heteromorphic ellipse.

The formula for the heteromorphic elliptic coefficient shows that this coefficient $\left(T_{h}\right)$ is a function only of the heteromorphic elliptic compression coeffi- 
cient $(\theta)$. In the interval of $[0.01,100]$, a series of $\theta$ values are given, and elliptic coefficients $\left(T_{h}\right)$ can be calculated by using a numerical integration method. A series of corresponding $T_{h}$ values are obtained, and the variation between the heteromorphic elliptic coefficients $\left(T_{h}\right)$ and compression coefficients $(\theta)$ can be determined, as shown in Figure 3. Under the same conditions, the variation relationships of elliptic coefficients are also given for comparison with the heteromorphic ellipse.

The results from calculation coupled with Figure 3 indicate that the heteromorphic elliptic coefficient of the Standard-type heteromorphic ellipse with equal width and height $(\theta=1)$ is $T_{h}=3.191$, is $1.58 \%$ larger than the elliptic coefficient $(=\pi)$ under the same conditions. Further analysis shows that the ratio of the area coefficient of the Standard-type heteromorphic ellipse to the heteromorphic elliptic coefficient is 0.249 . This value is only $0.31 \%$ lower than the ratio of these items in a circle, which shows that the area of a Standard-type heteromorphic ellipse is largest in the case of a certain perimeter value. In Figure 3, the elliptic coefficients (dashed line) are symmetric about the compression coefficient $\theta=1$ in logarithmic coordinates. For a given aspect ratio, the heteromorphic elliptic coefficient of the $\mathrm{H}$-type (compression coefficient $0<\theta<1$ ) is slightly larger than that of the $\mathrm{W}$-type $(\theta>1)$. When $\theta<0.1$, the relative difference between the heteromorphic elliptic coefficient and the elliptic coefficient is less than $5.0 \%$, and when $\theta>10$, the relative difference is less than $1.2 \%$.

\subsection{Radius of Curvature}

In principle, the curvature indicates the bending degree of a curve at a certain point, and the curvature is greater for a greater value of the bending degree of the curve. The reciprocal of the curvature is the radius of curvature. The radius of curvature is also used to describe the bending degree of a curve at a certain point, and the radius of curvature is smaller for greater values of the bending degree of the curve. The radius of curvature of the heteromorphic ellipse is closely related to the size, and the heteromorphic ellipse and the radius of the

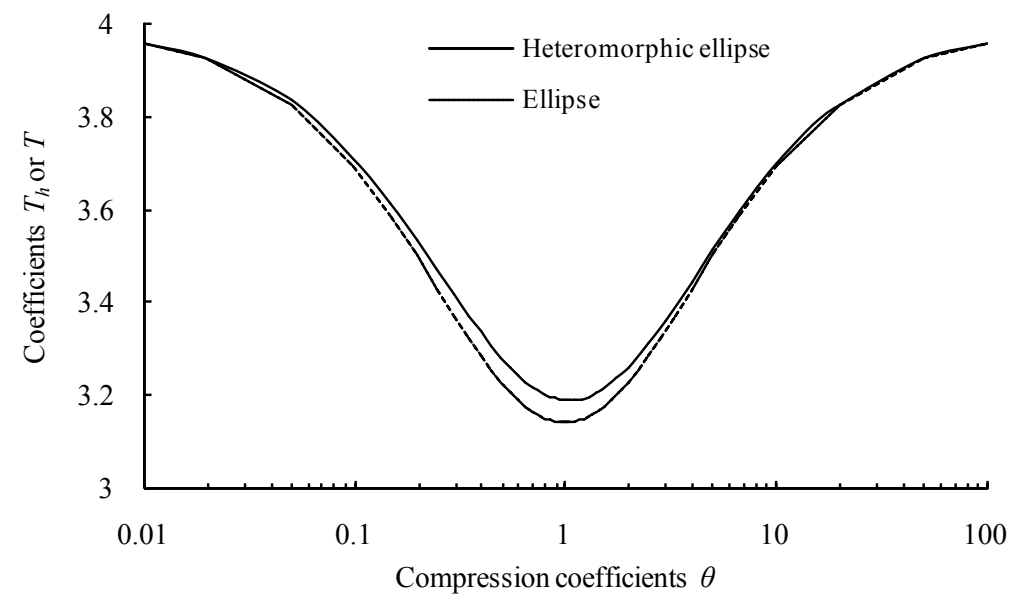

Figure 3. Comparison of heteromorphic ellipse coefficients and ellipse coefficients. 
corresponding point of the curve is larger for smaller values of the bending degree of the curve. To facilitate the analysis of the variation rule of the curvature radius of a heteromorphic ellipse, the Standard-type heteromorphic ellipse with $2 a=2 b=1$ is considered, and by Equation (6), the corresponding equation for a heteromorphic ellipse can be given as

$$
y^{2}=-\frac{\mathrm{e}}{4} x \ln (x)
$$

The first derivative is given as

$$
y^{\prime}=-\frac{\mathrm{e}}{8 y}[1+\ln (x)]
$$

The second derivative is given as

$$
y^{\prime \prime}=-\frac{\mathrm{e}+8 x y^{\prime 2}}{8 x y}
$$

The formula for radius of curvature is

$$
\rho=\frac{\left(1+y^{\prime 2}\right)^{3 / 2}}{\left|y^{\prime \prime}\right|}
$$

The definition of the domain is $0 \leq x \leq 1,-0.5 \leq y \leq 0.5$. Considering the symmetry of the heteromorphic ellipse, Figure 4 shows a plot of the half-curves of $y, y^{\prime}, y^{\prime \prime}$, and $\rho$ for analysis.

In Equation (18) and Equation (19), and Figure 4, it is clear that the heteromorphic ellipse $y=f(x)$ has first and second order continuous derivatives on the interval $(0,1)$. It is known from higher mathematics that both the curves of the heteromorphic ellipse and the first derivative are smooth.

From Equation (20) and Figure 4, it is apparent that the radius of curvature of the heteromorphic ellipse on the interval $[0,1]$ is continuous and presents a continuous variation over a"2" shape with $x$. In other words, as $x \rightarrow 0$, the radius of curvature $\rho \rightarrow \infty$, and with a gradual increase in $x, \rho$ decreases rapidly, and at $x=$ $0.043, \rho$ quickly reaches a minimum value of 0.248 . With further increases in $X, \rho$ gradually increases, and at $x=0.678, \rho$ reaches a maximum of 1.646. Subsequently, $\rho$ gradually decreases with increasing $x$ at $X=1$ and $\rho=0.340$.

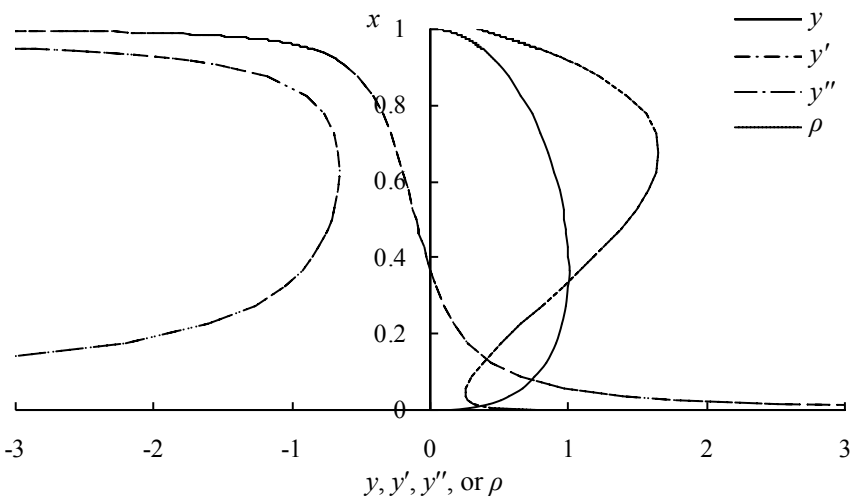

Figure 4. The half-curves of heteromorphic ellipse $y, y^{\prime}, y^{\prime \prime}$, and $\rho$ with $x$. 
Note that as the height, width, and compression coefficient of the heteromorphic ellipse vary, the radius of curvature changes continuously following a " 2 " shape, but the value of the radius of curvature and the position of the extreme point will change correspondingly.

\section{Geometric Properties of the Heteromorphic Elliptical Rotating Body}

\subsection{Rotating Body Volume}

Using definite integral, the volume of the body generated by revolving the heteromorphic ellipse (Equation (6)) through an angle of 360 degree around the $x$ axis (Symmetry axis) is

$$
V=\pi \int_{0}^{2 a} y^{2} \mathrm{~d} x=2 \pi \mathrm{e} a b^{2} \int_{0}^{2 a}-\frac{x}{2 a} \ln \left(\frac{x}{2 a}\right) \mathrm{d}\left(\frac{x}{2 a}\right)
$$

By performing variable substitution so that $x / 2 a=\zeta$, the upper limit of the integral becomes 1, (An integral table can be used to evaluate $\left.\int_{0}^{1} \zeta \ln \zeta \mathrm{d} \zeta=-1 / 4\right)$ and the result is given as

$$
V=-2 \pi \mathrm{e} a b^{2} \int_{0}^{1} \zeta \ln (\zeta) \mathrm{d} \zeta=\frac{\pi \mathrm{e}}{2} a b^{2}
$$

This value is $1.94 \%$ larger than the volume of the ellipsoid.

\subsection{Surface Area of Rotating Body}

Using definite integral, the surface area of the body generated by revolving the heteromorphic ellipse, given in Equation (6), through an angle of 360 degree around the $x$ axis is expressed as

$$
S_{a}=2 \pi \int_{0}^{2 a} y \sqrt{1+y^{\prime 2}} \mathrm{~d} x
$$

Substituting Equation (6) and Equation (15) into Equation (23), setting $x / 2 a=$ $\zeta$, and noting that $b / a=\theta$, the upper limit of the integral becomes 1 and the result is given as

$$
S_{a}=8 \pi a b \int_{0}^{1} \sqrt{-\frac{\mathrm{e}}{4} \zeta \ln \zeta} \sqrt{1-\frac{\mathrm{e} \theta^{2}}{16} \frac{(1+\ln \zeta)^{2}}{\zeta \ln \zeta}} \mathrm{d} \zeta=\lambda(\theta) 4 \pi a b
$$

where

$$
\lambda(\theta)=2 \int_{0}^{1} \sqrt{-\frac{\mathrm{e}}{4} \zeta \ln \zeta} \sqrt{1-\frac{\mathrm{e} \theta^{2}}{16} \frac{(1+\ln \zeta)^{2}}{\zeta \ln \zeta}} \mathrm{d} \zeta
$$

$\lambda(\theta)$ is defined as the "surface area coefficient" of the rotating body of heteromorphic ellipse around the $x$ axis. Taking a series of values in interval $(0.1 \leq \theta$ $\leq 10.0$ ) and integrating Equation (25) by numerical integration method, the results are plotted using the "+" points as shown in Figure 5.

According to Figure 5 and the calculations, the surface area coefficient of the rotating body of heteromorphic ellipse around the $x$ axis shows a monotonic rising trend from 0.800 to 5.161 in interval $(0.1 \leq \theta \leq 10.0)$. The surface area 


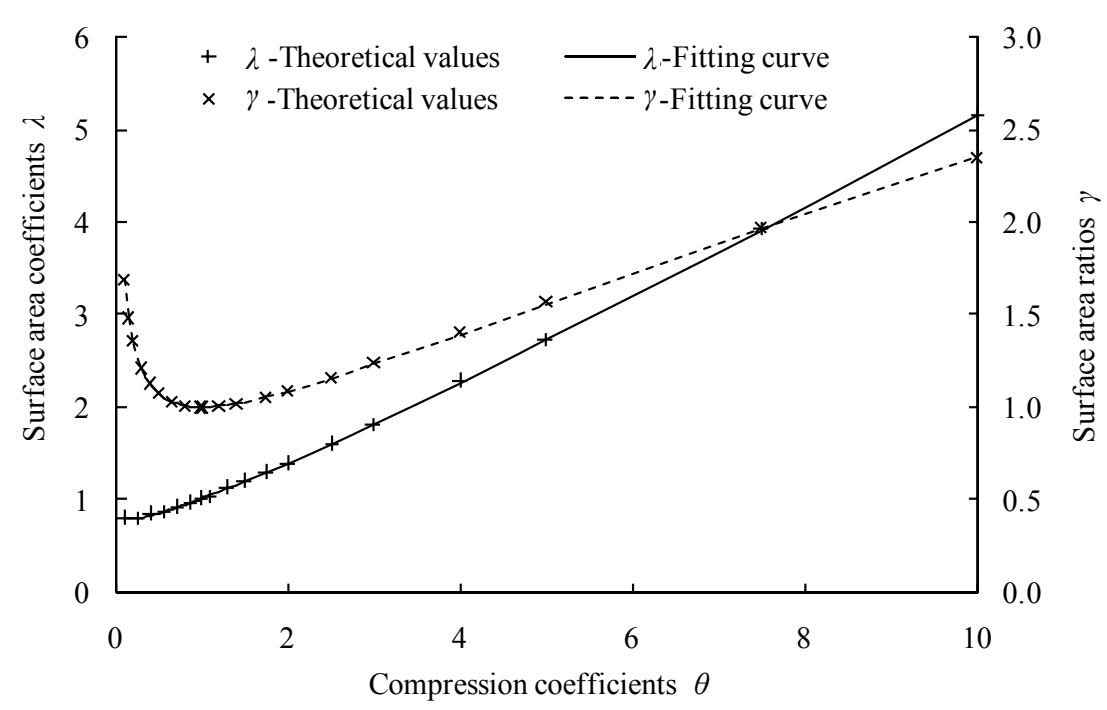

Figure 5. Variation curves of the surface area coefficient and the surface area ratio of the rotating body with the compression coefficient.

coefficient of the rotating body of Standard-type heteromorphic ellipse around the $x$ axis $(\theta=1)$ is $\lambda(1)=1.0212$, indicating that the surface area of this rotating body is $2.12 \%$ larger than that of a sphere with radius $r(=a=b)$. The fitting curve equation of the surface area coefficient $\lambda(\theta)$ is given as

$$
\lambda=0.3681 \theta^{1.099}+0.6559 \theta^{-0.0806}
$$

The correlation coefficient $\mathrm{R}^{2}=0.9999$, and the absolute values of the relative errors are less than $1.02 \%$.

\subsection{Surface Area Ratio of Rotating Body}

Definition of the "surface area ratio" of the rotating body of heteromorphic ellipse around the $x$ axis is as follows. Under a same volume of the rotating body, the ratio of $S_{a}$, which is the surface area of the rotating body of heteromorphic ellipse around the $x$ axis with different $\theta$, to $S_{a 0}$, which is the surface area of the rotating body of Standard-type heteromorphic ellipse around the $x$ axis with $\theta=$ 1 , is the surface area ratio $\gamma(\theta)$ of the rotating body of heteromorphic ellipse around the $x$ axis is given as

$$
\gamma=\frac{S_{a}}{S_{a 0}}=\frac{\lambda(\theta)}{\lambda(1) \theta^{1 / 3}}
$$

The results of calculation are shown as the " $x$ " points in Figure 5.

According to Figure 5 and the calculations, in interval $(0.1 \leq \theta \leq 10.0)$, the surface area ratio of the rotating body of heteromorphic ellipse around the $x$-axis presents a curve change rule in the shape of " $\sqrt{ }$ ". The minimum surface area ratio $\gamma_{\min }=0.999977$ appears at compressibility $\theta=1.011561$, i.e., the surface area ratio of the rotating body of Standard-type heteromorphic ellipse around the $x$ axis, where $\gamma_{0}=1$ is not the minimum value of surface area ratio. On the left side of the minimum surface area ratio $\gamma_{\min }, \gamma(\theta)$ increases with decrease of compres- 
sion coefficient, whereas on the right side, $\gamma(\theta)$ increases with increase of compression coefficient. The fitting curve equation of surface area ratio $\gamma(\theta)$ is given

$$
\gamma=0.334 \theta^{0.7958}+0.671 \theta^{-0.3911}
$$

The correlation coefficient $\mathrm{R}^{2}=0.9995$, and the absolute values of the relative errors are less than $0.98 \%$.

As per the " $\sqrt{ }$ " curve Equation (28) of surface area ratio of the heteromorphic elliptical rotating body, the compression coefficient of the corncob is about 0.25 , and its surface area ratio is 1.26 . Further, the compression coefficient of sunflower disk is about 5.50, and its surface area ratio is 1.64 , whereas the compression coefficients of apple, pears, and other fruits are $0.80-1.25$, and their surface area ratios are proximate to the minimum value of 1.00 (less than 1.01).

\section{Case Analysis and Application Prospects}

\subsection{Case Analysis}

Taking a traffic tunnel as a sample case, an application and comparative analysis have been carried out. According to the relevant provisions of China's highway tunnel design code, Wang have given the design limits (maximum size of 1025 $\mathrm{cm} \times 500 \mathrm{~cm}$ ) of highway tunnels and the inner contour design of a three-heart-curve round tunnel with a unidirectional double-lane design speed of $80 \mathrm{~km} / \mathrm{h}$, as shown in Figure 6 [11]. Table 1 shows the parameters of the three-heart-curve round tunnel.

According to Figure 6 and Table 1, in addition to the inverted arch of backfill area, a three heart curve round tunnel is composed of three arc centers, namely, crown arch, right arch, and left arch. It is composed of a crown arch and two side arches.

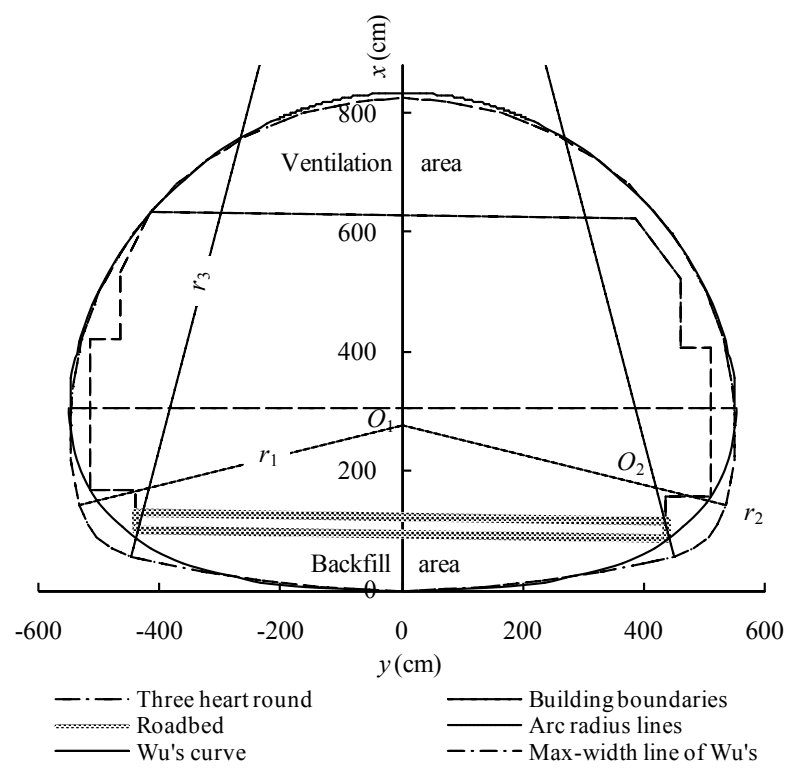

Figure 6. Design of heteromorphic elliptical section for a tunnel project. 
Table 1. Parameters of the three-heart-curve round tunnel.

\begin{tabular}{cccccc}
\hline \multirow{2}{*}{ Center Number } & Radius $(\mathrm{cm})$ & \multicolumn{2}{c}{ Center Coordinates } & \multirow{2}{*}{ Center Angles } & Remarks \\
\cline { 3 - 4 } & & $x(\mathrm{~cm})$ & $y(\mathrm{~cm})$ & & \\
\hline 1 & 550 & 275 & 0 & $2 \times 103^{\circ} 45^{\prime} 24^{\prime \prime}$ & Crown arch \\
2 & 120 & 172.7 & 417.7 & $61^{\circ} 50^{\prime} 53^{\prime \prime}$ & Right arch \\
3 & 120 & 172.7 & -417.7 & $61^{\circ} 50^{\prime} 53^{\prime \prime}$ & Left arch \\
- & 1800 & 1800 & 0 & $2 \times 14^{\circ} 23^{\prime} 43^{\prime \prime}$ & Invert arch \\
\hline
\end{tabular}

If the heteromorphic ellipse (Wu's curve) is used as the inner contour of the tunnel and the structural boundaries are completely contained, the optimal design can be implemented according to the minimum cross-sectional area of the tunnel to obtain the maximum width $W=1100 \mathrm{~cm}$ and the maximum height $H$ $=835 \mathrm{~cm}$ for the cross section of the tunnel inner contour. Figure 6 shows the inner contour design for a heteromorphic elliptical tunnel.

The main advantages and disadvantages of the inner contour of a heteromorphic elliptical tunnel and the three-heart-curve round cross section have been compared and analyzed as given below.

1) The inner contour of a heteromorphic elliptical tunnel requires only two design parameters, whereas the three-heart-curve round cross section requires four independent parameters. The former is a trivial one for design and optimization, easy to implement, and enables error control.

2) Substituting Formula (10), $W=1100 \mathrm{~cm}$ and $H=835 \mathrm{~cm}$ into Formula (8), the inner contour area of the heteromorphic elliptical tunnel is obtained as

$$
S=\mu(2 a)(2 b)=0.795 W H=73.05 \mathrm{~m}^{2},
$$

and following the calculations from a geometric figure, the cross-sectional area of the three-heart-curve round tunnel is $74.04 \mathrm{~m}^{2}$. The former can save $1.34 \%$ of the earthwork of excavation for a long-distance tunnel.

3) From the compression coefficient $\theta=W / H=1.317$, the heteromorphic elliptic coefficient $T_{h}=3.200$ can be calculated. Then the inner contour perimeter of the heteromorphic elliptical tunnel is $L=T_{h}(W+H) / 2=30.96 \mathrm{~m}$, and as calculated from the geometric figure, the cross-sectional perimeter of the three-heart-curve round tunnel is $31.56 \mathrm{~m}$. The former can save $1.88 \%$ in tunnel support and lining construction material, which is beneficial for reducing project investment.

4) The maximum height of the inner contour of the heteromorphic elliptical tunnel is $H=835 \mathrm{~cm}$ and the maximum height of the cross section of the three-heart-curve round tunnel is $H=825 \mathrm{~cm}$. The maximum widths of the two tunnel cross sections are equal. Figure 6 shows that the top arch of the inner contour of the heteromorphic elliptical tunnel is slightly higher, which helps to increase tunnel ventilation and to withstand the pressure at the top of the tunnel. Moreover, the inner contour of the heteromorphic elliptical tunnel overcomes the convexities of the arch footing and the cross section of the three-heart-curve 
round tunnel, which helps to save the quantity of backfill concrete required for the inverted arch.

5) There is a continuous second-order derivative in the inner contour of the heteromorphic elliptical tunnel, and the radius of curvature shows a continuous change alonga" 2 " shape with the height of the cross section. However, the three values of the radii of curvature in the cross section of the three-heart-curve round tunnel is apparently distorted at the connection point (tangent point).

\subsection{Application Prospects}

In human civilization, patterns develop following two basic geometric shapes, viz. one is straight-line and rectangular, and the other is an ellipse (including circle and circle arcs). The straight line makes things easy to reconcile with the shortest distance, whereas the ellipse gives a flexible and easily moving feeling, both physically and psychologically. For centuries, designers have been limited by this "round or square" classification of thinking. Custom-developed hyperelliptical shapes have not been used until Piet Hein's design [4]. The discovery of the equation of the heteromorphic ellipse (Wu's curve) undoubtedly have expanded our scope to choose graphic forms.

\section{- Tunnel}

The tunnel is an engineering structure buried in an earth stratum and is a form of human use of underground space. For more than a century, due to the lack of suitably shaped curves, the inner contours of existing tunnels were mainly characterized by three-centered, four-centered, and five-centered circles and shapes created by arc splicing. Taking a four-centered circular tunnel cross section as an example, there are five independent variables: radius $r_{1}, r_{2}, r_{3}$, and the central angles $\varphi_{1}, \varphi_{2}$ [12]. The inner contour of a tunnel formed by multiple circular arcs can provide continuity and smoothness of the curve at the connection point (tangent point).However, the first derivative is continuous and not smooth, but the second derivative, where the curvature distortion and stress concentration phenomena occur, is discontinuous [13]. From the analysis as shown in Section 4.5, it is obvious that the inner contour of a heteromorphic elliptical tunnel has obvious advantages as compared with the cross section created by multiple arc splicing. The inner contour of a heteromorphic elliptical tunnel and its derivatives has better continuity, differentiability, smoothness, and integrity.

\section{- Liquid transport tank}

The cross section of the common liquid transport tank is a circle, an ellipse, or a hyper-elliptical shape between an ellipse and a rectangle. If the tank is liquid-filled, the position of the cross-sectional centroid (center of gravity) is the same as the median line. At the same width and height for heteromorphic ellipses and ellipses, the centroid position of a cross section of the heteromorphic ellipse is $7.05 \%$ lower than the median line (the ellipse centroid), which can greatly improve the safety of liquid tanks in vehicle driving, especially on curves. The heteromorphic ellipse is wider in the lower part and flatter at the bottom than a circle or an ellipse, and the required bracket is short, has a small force to 
resist, and easy to fix. Therefore, the heteromorphic ellipse has a good stability on a vehicle chassis, and it is an ideal cross-sectional shape for a liquid transport tank.

\section{- Airplanes and submarines}

The cross sections of common airplanes and submarines are circular. Under the same width and height conditions, the lower space of a heteromorphic ellipse is larger than that of a circle, which increases the available space for equipment installation and cargo loading, and the center of gravity of airplanes can be lowered accordingly, which is beneficial for flight stability. For submarines, the inverted cross section of the heteromorphic ellipse is used, with which the upper part of the submarine is wider than the circular cross section. Hence, it is easy to decorate the offices and living spaces, and the lower part of the submarine can be used for equipment installation and power. The center of buoyancy of an inverted heteromorphic ellipse is $7.05 \%$ higher than that of a circle, which is favorable for keeping submarines in a stable equilibrium state. This is a new field worth studying by researchers in related disciplines and applications.

\section{- Bridges, buildings, furniture, and handicrafts}

Like the superellipse, the heteromorphic ellipse (Wu's curve) can also be used in the design of bridge piers, square buildings, furniture, and handicrafts. Examples include beds, tables, silverware, dishes, vases, candlesticks, casseroles, soup pots, teapots, and wine glasses.

It has to be emphasized that the heteromorphic ellipse is invented based on the isoconcentration-curve formula as the solution of a simplified advection-diffusion transport of a constant point source. If considering the pedicle as a point source during fruit growth, it is intuitively obvious that the shape of the fruit such as the corn cob, apple, persimmon, pineapple and pear, is very similar or close to the heteromorphic ellipse: their shape is not only affected by the type of species and natural conditions (climate and sunlight, for example), but also constrained by the isoconcentration condition during nutrition transport. Therefore, heteromorphic ellipse can also be a research object of great concern for the botanical research community.

\section{Conclusions}

1) An equation for anew heteromorphic elliptic (Wu's curve) with two parameters for $x$-symmetry is defined. In the heteromorphic ellipse, the corresponding height of the maximum width and the height of the centroid position are given. The shapes of heteromorphic ellipses are classified into a heteromorphic ellipse, which belongs to the $\mathrm{H}$-type with a compression coefficient of $0<\theta=b / a$ $<1$, standard-type with $\theta=1$, and the W-type with $\theta>1$.

2) The area formula can be given as, the area of a heteromorphic ellipse is equal to the product of the area coefficient, height, and width, viz. $S=\mu(2 a)(2 b)$. Besides, the perimeter theorem of the heteromorphic ellipse can be stated as, perimeter of the heteromorphic ellipse is equal to the product of the heteromorphic elliptic coefficient and the sum of the half-height and half-width of the he- 
teromorphic ellipse, viz. $L=T_{h}(a+b)$.

3) It has been shown that the first- and second-order derivatives of the heteromorphic ellipse are continuous, indicating that both the heteromorphic ellipse and its first-derivative curve are smooth, and the variation in the radius of curvature with the height coordinate $x$ follows a continuous " 2 " shape. Thereafter, the geometric properties of the rotating body are proposed.

4) The case analysis shows that, when compared with the three-heart-curve round cross section, the inner contour of a heteromorphic elliptical tunnel has the advantages of fewer design parameters, a continuous radius of curvature, smaller tunnel excavation area, shorter circumference of support and lining, higher cavern (which favors ventilation), and smaller backfilling quantities.

5) The analysis also shows that the heteromorphic ellipse and its derivatives have good continuity, differentiability, smoothness, and integrity, which give these shapes broad application prospects in the design of tunnels, liquid transport tanks, planes, submarines, bridges, buildings, furniture, and crafts.

\section{Funding}

This research is supported by the National Natural Science Foundation of China (Grant No. 51379097, No. 50979036).

\section{Acknowledgements}

We thank International Science Editing

(http://www.internationalscienceediting.com) for editing this manuscript.

\section{Conflicts of Interest}

The author declares no conflicts of interest regarding the publication of this paper.

\section{References}

[1] Besant, W.H. (2009) Conic Sections: Treated Geometrically. Gutenberg eBook. http://www.gutenberg.org/files/29913/

[2] Sokolov, D.D. (2001) "Lamé Curve”, in Hazewinkel, Michiel, Encyclopedia of Mathematics. Springer-Verlag New York, Inc.

[3] Lockwood, E.H. (1961) A Book of Curves. Cambridge University Press, Cambridge. https://doi.org/10.1017/CBO9780511569340

[4] Gardner, M. (1977) "Piet Hein's Superellipse”, Mathematical Carnival. A New Round-Up of Tantalizers and Puzzles from Scientific American. Vintage Press, New York. https://www.researchgate.net/publication/285702127 Piet Hein's superellipse

[5] Gardner, M. (1997) The Last Recreations: Hydras, Eggs, and Other Mathematical Mystifications. Springer-Verlag New York, Inc. https://xueshu.baidu.com/usercenter/paper/show?paperid=f36fdde99ef64e9e25d9de 0e26ebf76b\&site $\% 20=\% 20 x u e s h u$ se

[6] Jürgen, K. (2000) Egg Curves and Ovals. 
http://www.mathematische-basteleien.de/eggcurves.htm

[7] Gu, C.H. (1992) Mathematics Dictionary. 1st Edition, Shanghai Lexicographical Publishing House, Shanghai. (In Chinese)

[8] Li, W.H. (1972) Differential Equations of Hydraulic Transients, Dispersion and Ground Water Flow. Prentice Hall, Englewood Cliffs.

[9] Wu, Z.H. and Jia, H.Y. (2009) Analytic Method for Pollutant Mixing Zone in River. Advances in Water Science, 20, 544-548. (In Chinese). http://www.en.cnki.com.cn/Article en/CJFDTOTAL-SKXJ200904015.htm

[10] Wu, Z.H., Wu, W. and Wu, G.Z. (2011) Calculation Method of Lateral and Vertical Diffusion Coefficients in Wide Straight Rivers and Reservoirs. Journal of Computers, 6, 1102-1109.

https://dblp.uni-trier.de/search?q = Calculation + Method + of + Lateral + and + Vertical + Diffusion https://doi.org/10.4304/jcp.6.6.1102-1109

[11] Wang, X.D. (2009) Preliminary Research on the Standardization of Highway Mountain Tunnels (Chinese). Master's D. Thesis, Chang'an University, Xi'an.

[12] Zhang, J.G. (2004) The Study on the Optimized Method of Highway Tunnel Support Structure Design. Shanxi Science \& Technology of Communications, 5, 59-60. (In Chinese) http://en.cnki.com.cn/Article en/CJFDTOTAL-SXJT200405025.htm

[13] Naggar, H.E. and Hinchberger, S.D. (2012) Approximate Evaluation of Stresses in Degraded Tunnel Linings. Soil Dynamics \& Earthquake Engineering, 43, 45-57. https://doi.org/10.1016/j.soildyn.2012.07.016 